\title{
Surface Plasmon Modes in Long Chains of Au Nanoparticles
}

Pavel Potapov ${ }^{1,2}$, Martin Mayer ${ }^{3}$, Axel Lubk $^{2}$, Darius Pohl ${ }^{2,4}$, Johannes Schultz ${ }^{2}$, Tobias A. F. König ${ }^{3}$ and Andreas Fery ${ }^{3}$

1. Leibniz Institute for Solid State and Materials Research (IFW), Dresden, Germany.

2. Technical University of Dresden, Department of Physics, Dresden, Germany.

3. Leibniz Institute of Polymer Research (IPF), Dresden, Germany.

4. Technical University of Dresden, Center for Nanoanalysis, Dresden, Germany.

Recent advances in the nano-synthesis allow creating ensembles of nanoparticles with well-defined geometry suitable for selectively confining and transferring of optical signals in plasmonic devices. In particular, highly ordered chains of metallic nanoparticles ("plasmonic polymers") are currently considered as bottom-up assembled plasmonic wave guides. Probing and understanding the details of the plasmon excitations in such ensembles are crucial for designing nanodevices with target plasmonic properties, such as wave guiding. The long chain limit is especially important for applications while has not been studied in detail previously.

Here we present a result of EELS STEM study of linear chains with varying number of Au nanoparticles carried out on the monochromated probe-corrected FEI Titan ${ }^{3}$ microscope. The spectrum-images were acquired with a beam current $200 \mathrm{pA}$, dwell time $25 \mathrm{~ms}$ and the energy resolution of $120 \mathrm{meV}$ (FWHM). For each dataset, 4-7 runs of acquisition were performed followed by the summation of the data cubes after correction for spatial drift and energy instabilities. The zero-loss peak was removed with the subpixel precision using the reference vacuum profile. Finally, the magnitude of all distinct peaks in the low-loss spectra was fitted with the NLLS procedure giving rise to maps of the probability for given plasmon excitations.

The principle dipolar surface plasmon mode with an excitation energy of about $2.2 \mathrm{eV}$ is visualized as a halo around the particles surfaces independent of the chain length (Fig. 1). On top of that, a coupling among particles results in additional plasmon modes at lower energies [1]. We observe the standing waves with the order of harmonics $L=1,2$ and 3 as seen from the examples in Fig.1, 2. These modes are analogous to those observed previously on the surface of nanorods [2] although, in our case, the Au particles were not in the direct contact with each other, thus all correlations could occur through the electromagnetic fields only. To understand the dispersion relation in the observed modes we consider a simple discrete dipoles model that accounts for the interaction coupling between nearest neighbors in the chain. This model shows a reasonable agreement with the experimentally observed excitation energies (Fig.3) and allows predicting the crucial properties of long chains analytically.

Accordingly, in longer chains, all modes are red shifted to the intraband dissipative region, therefore harmonics with low $L$ might eventually disappear. When the number of particles exceeds 10 , the high- $L$ modes mix up forming a kind of continuous band where individual harmonics are indistinguishable [4].

References:

[1] S. J. Barrow et al, Nanoletters, 14 (2014), p. 3799.

[2] M. Mayer et al, Nanoletters, 15 (2015), p. 5427. 
[3] U. Hohenester, Computer Physics Communications, 185 (2014), p.1177.

[4] PP acknowledges funding from DFG “Zukunftskonzept” (F-003661-553-Ü6a-1020605). AL received funding ERC under the Horizon 2020 program (grant 715620).

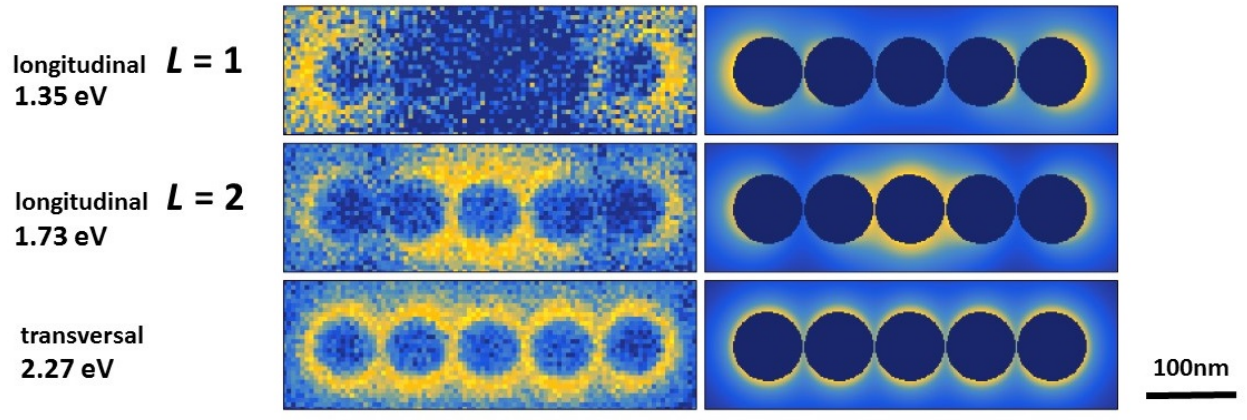

Figure 1. Excitation probability maps for the various surface plasmon modes in the chain of $5 \mathrm{Au}$ nanoparticles. The lower map corresponds to the principal transverse mode while the upper maps are associated with the longitudinal standing waves with harmonics $L=\mathbf{1}$ and $\mathbf{2}$. The experimental maps are in the left and maps simulated with Matlab MNPBEM toolbox [3] are in the right.

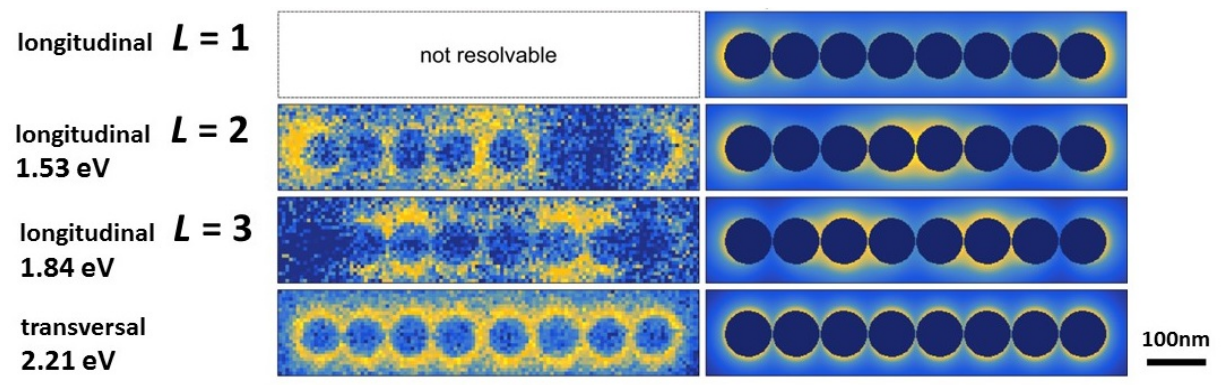

Figure 2. Excitation probability maps for the various surface plasmon modes in the chain of $\mathbf{8} \mathrm{Au}$ nanoparticles. The lower map corresponds to the principal transverse mode while the upper maps are associated with the longitudinal standing waves with harmonics $L=\mathbf{2}$ and $\mathbf{3}$. The experimental maps are in the left and maps simulated with Matlab MNPBEM toolbox [3] are in the right.

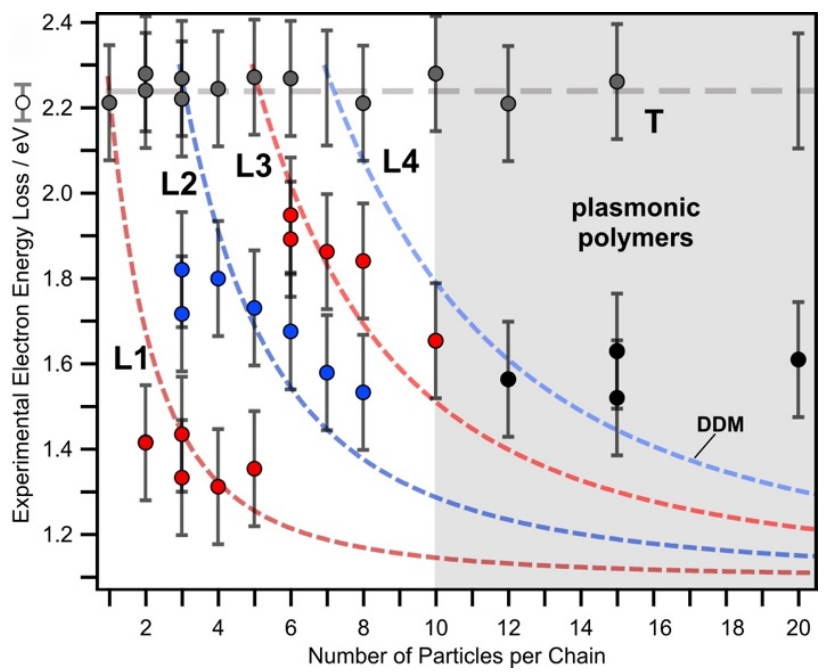

Figure 3. Experimental energies for various surface plasmon modes and those calculated with the Discrete Dipole Model (dashed lines). Transverse mode (T) and longitudinal harmonics (L) are shown. 